Supporting information

\title{
Boron Modified Defect-Rich Molybdenum Disulfide Nanosheets: Reducing Nonspecific Adsorption and Promoting a High Capacity for Isolation of Immunoglobulin $\mathrm{G}$
}

Xi-Ming Wang, Zheng-Jie Hu, Peng-Fei Guo, Ming-Li Chen* and Jian-Hua Wang*

Research Center for Analytical Sciences, Department of Chemistry, College of

Sciences, Northeastern University, Box 332, Shenyang 110819, China

*Corresponding author.

*E-mail address: chenml@mail.neu.edu.cn. (M.L.Chen), jianhuajrz@mail.neu.edu.cn (J.H. Wang).

Tel: +86 24 83688944; Fax: +86 2483676698 


\section{Experimental part}

Chemical reagents. Hexaammonium heptamolybdate tetrahydrate $\left(\left(\mathrm{NH}_{4}\right)_{6} \mathrm{Mo}_{7} \mathrm{O}_{24} \cdot 4 \mathrm{H}_{2} \mathrm{O}\right)$, thiourea, imidazole, Coomassie brilliant blue G-250, sodium hydroxide, hydrochloric acid, methanol, ethanol, acetic acid, phosphoric acid, boric acid, $\mathrm{NH}_{3} \cdot \mathrm{H}_{2} \mathrm{O}$ and sodium chloride were purchased from Sinopharm Chemical Reagent Co. Ltd. (Shanghai, China). 5-carboxybenzoboroxole (CBX) was ordered from AK Scientific, Inc. (USA). Tris(hydroxymethyl)methyl aminomethane (Tris), sodium dodecyl sulfate (SDS), cetane trimethyl ammonium bromide (CTAB), 2-[4-(2-hydroxyethyl)piperazin-1-yl]ethanesulfonic acid (HEPES), N-hydroxy succinimide (NHS), 1-(3-Dimethylaminopropyl)-3-ethylcarbodiimide hydrochloride (EDC), and DL-thioctic acid, also named alpha lipoic acid (LA) were obtained from Aladdin Bio-Chem Technology Co., Ltd. (Shanghai, China). The reagents are at least of analytical reagent grade and are used without further treatment or purification. L-lysine (Lys), immunoglobulin G from human serum (IgG, I4506, Mr 150.0 kDa), concanavalin A from Jack bean (ConA, L7647, Mr 102.0 kDa), cytochrome c (Cyt-c, 30398, Mr 12.4 kDa), lysozyme from chicken egg white (LZM, 62970, Mr 14.3 kDa) and bovine serum albumin (BSA, A3311 Mr 66.7 kDa) are purchased from Sigma-Aldrich (St. Louis, USA). $\gamma$-globulin from bovine milk ( $\gamma$-Glo, G0034 Mr $150.0 \mathrm{kDa}$ ) is obtained from TCI (Tokyo Kasei Kogyo Co. Ltd., Japan). The protein molecular weight marker (broad, 3452, Dalian, China, Takara Biotechnology Company) is a mixture of nine purified proteins ( $\mathrm{Mr}$ in $\mathrm{kDa}$ : myosin, 200; $\beta$-galactosidase, 116; phosphorylase B, 97.2; serum albumin, 66.4; ovalbumin, 44.3; 
carbonic anhydrase, 29; trypsin inhibitor, 20.1; lysozyme, 14.3; aprotinin, 6.5). Human serum samples are provided by healthy volunteer from the Hospital of Northeastern University, China.

Instrumentations. Scanning electron microscopic (SEM) images were carried on a LEO1430VP Scanning Electron Microscope (LEO, Germany). Transmission electron microscopy (TEM) images were obtained from a JEM-1011 (JEOL, Japan) with an accelerating voltage of $100 \mathrm{kV}$. X-ray diffraction (XRD) patterns were performed on a PANalytical Empyrean diffractometer (PANalytical BV, Netherlands) with $\mathrm{Cu} \mathrm{K} \alpha$ radiation at $\lambda 1.54 \AA$. X-ray photoelectron spectroscopy (XPS) measurements were recorded by using a Thermo Scientific ESCALAB 250Xi electron spectrometer (Thermo Electron, America) with an $\mathrm{Al} \mathrm{K \alpha} 1486.6 \mathrm{eV}$ excitation source. Fourier transform infrared spectra (FT-IR) were carried out on a Nicolet 6700 spectrophotometer (Thermo Electron, USA) from 4000 to $400 \mathrm{~cm}^{-1}$ with $\mathrm{KBr}$ pellets. Zeta potential measurements were collected on a Nano ZS90 (Malvern, UK). The water contact angle was measured using a DSA25 (KRUSS, Germany). The circular dichroism (CD) spectra are recorded with a MOS-450 spectrometer/polarimeter (Biologic Science Instrument, France) by using a $0.1 \mathrm{~cm}$ quartz cell. The $\mathrm{pH}$ was adjusted by a PB-10 pH meter (Sartorius, Germany).

Operations of SDS-PAGE assay. For SDS-PAGE assay, all the sample aqueous solutions are mixed with loading buffer and boiling for $10 \mathrm{~min}$. Then the electrophoresis experiments are demonstrated on 5\% polyacrylamide stacking gel and $12 \%$ polyacrylamide separation gel at $90 \mathrm{~V}$ and $180 \mathrm{~V}$, respectively. The separation gel is stained with $0.1 \%(\mathrm{w} / \mathrm{v})$ Coomassie Brilliant Blue G-250, and destained with a mixture solution containing $7.5 \%(\mathrm{v} / \mathrm{v})$ acetic acid and $5 \%(\mathrm{v} / \mathrm{v})$ methanol. 

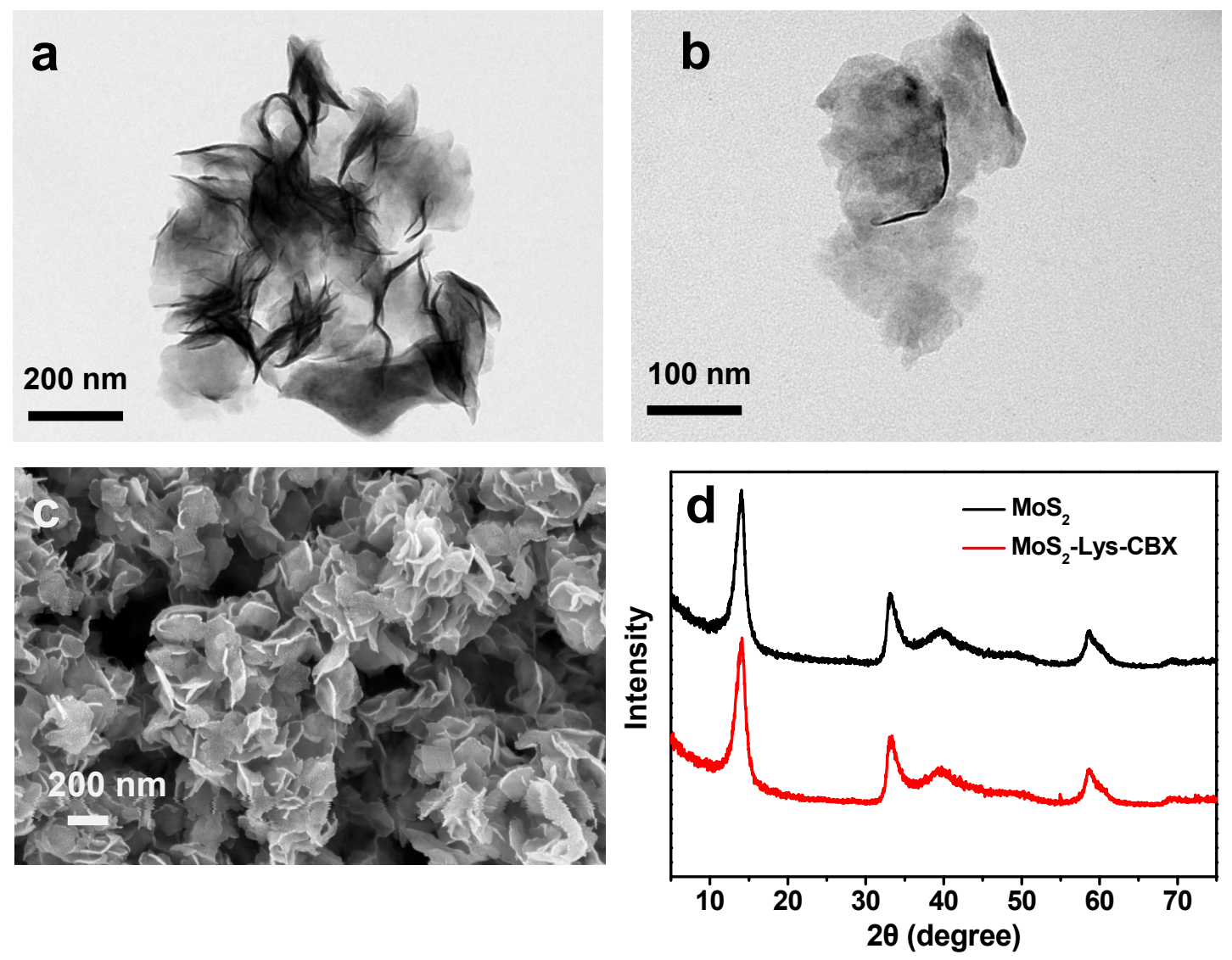

Figure S1. TEM images (a, b) and SEM image (c) of $\mathrm{MoS}_{2}$. XRD patterns (d) of $\mathrm{MoS}_{2}$ and $\mathrm{MoS}_{2}$-Lys-CBX composites. 

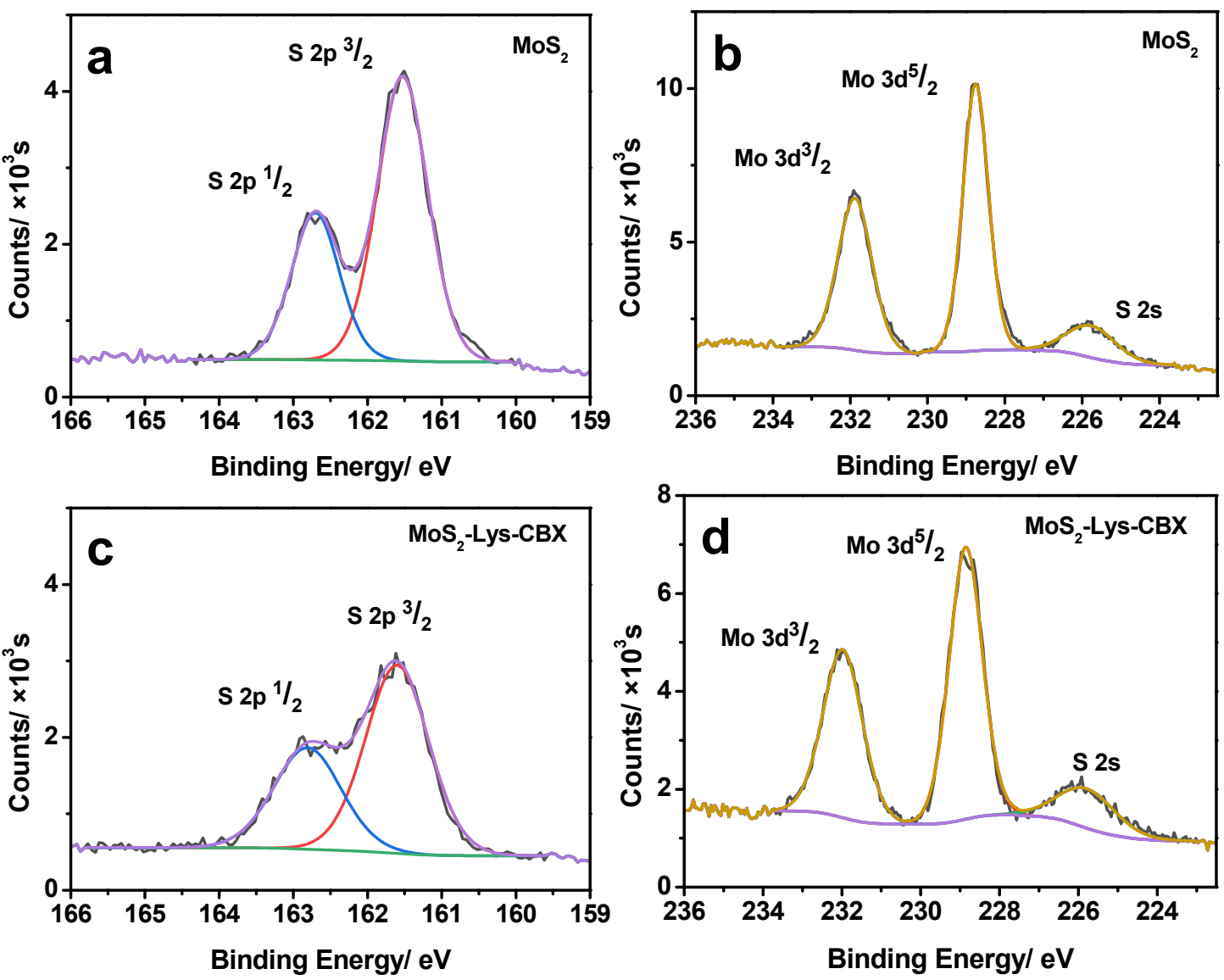

Figure S2. High-resolution XPS S 2p (a, c) and Mo 3d (b, d) peaks of $\mathrm{MoS}_{2}$ and $\mathrm{MoS}_{2}$-Lys-CBX composites, respectively. 


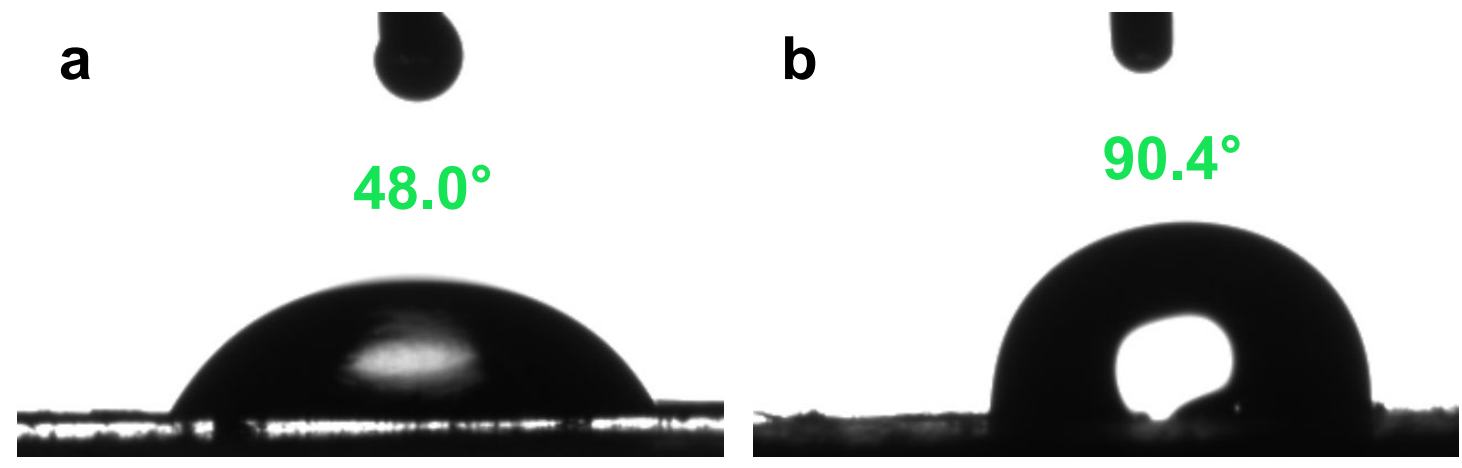

Figure S3. The contact angle of $\mathrm{MoS}_{2}$-Lys-CBX composites (a) and $\mathrm{MoS}_{2}$ (b). 


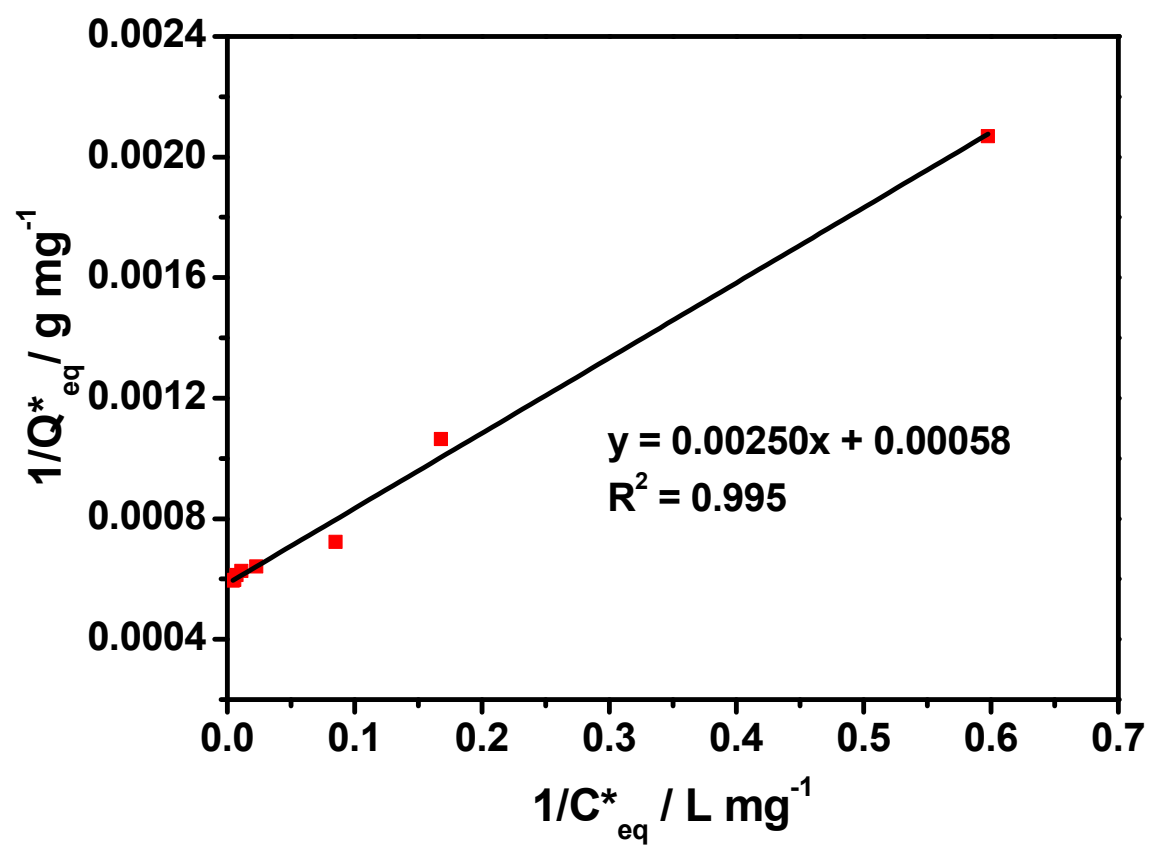

Figure S4. The plot of $1 / Q^{*}$ eq versus $1 / C^{*}$ eq. 